\title{
„Funktionelle Körperbeschwerden“: Klinisch relevante Leitlinien-Empfehlungen
}

Casper Roenneberg, Constanze Hausteiner-Wiehle, Peter Henningsen

\author{
Behandler erleben Patienten mit funktionellen Körperbeschwerden oft als \\ „schwierig“ - auch weil ihnen häufig das Wissen fehlt, wie sie mit dieser sehr \\ heterogenen Patientengruppe umgehen und diese behandeln sollen. Dieser \\ Beitrag soll Anwender bei diagnostischen und therapeutischen Entscheidungen \\ unterstützen und dadurch helfen, die Lebensqualität der Betroffenen sowie die \\ beiderseitige Behandlungszufriedenheit zu erhöhen.
}

\section{Hintergrund}

Unter Federführung der Deutschen Gesellschaft für Psychosomatische Medizin und Ärztliche Psychotherapie (DGPM) und des Kollegiums für Psychosomatische Medizin (DKPM) wurde die Leitlinie „Funktionelle Körperbeschwerden“ von der Leitliniengruppe (32 Fachgesellschaften, 2 Patientenvertretungen) nach den Kriterien der Arbeitsgemeinschaft wissenschaftlicher medizinischer Fachgesellschaften (AWMF) verabschiedet. Die Leitliniengruppe analysierte nach systematischer Literaturrecherche hierzu 3795 neue Originalarbeiten und 3345 Übersichtsarbeiten. Ziel war die Erstellung einer aktuellen, interdisziplinären, evidenzbasierten, anwenderfreundlichen und praxisnahen klinischen Leitlinie.

\section{Körperbeschwerden als Befindlich- keitsstörungen}

Körperbeschwerden sind generell häufig, präsentieren sich mit unterschiedlichen Symptomen und sind in ihrer Ausprägung bzw. in Ihrem Schweregrad verschiedenartig.

Viele Menschen berichten über individuell unterschiedlich belastende Körpersymptome wie Kraftlosigkeit, Schwindel, Übelkeit, Kopf- und/oder Gliederschmerzen, Verspannungen, Verdauungsbeschwerden und andere Symptome.

Vorübergehende „Befindlichkeitsstörungen“ haben keinen Krankheitswert per se, führen nur selten zu einem Arztbesuch und beeinträchtigen die Lebensqualität und Funktionsfähigkeit allenfalls kurzfristig und meist nur geringfügig.
In Studien berichten $82 \%$ des untersuchten gesunden Kollektivs über Körperbeschwerden, die sie innerhalb der letzten Woche zumindest leicht beeinträchtigten. $22 \%$ gaben mindestens eine Beschwerde an, die sie in den letzten 7 Tagen schwer beeinträchtigte [1].

Merke

Körperbeschwerden kommen häufig vor, führen nur selten zu einem Arztbesuch, beeinträchtigen die Funktionsfähigkeit allenfalls kurzfristig und geringfügig und haben nicht per se einen Krankheitswert; sie sind stattdessen Teil von Gesundheit.

\section{Begrifflichkeit „Funktionelle Körper- beschwerden"}

Unter „funktionellen Körperbeschwerden“ versteht man ein breites Spektrum an sehr verschiedenen Beschwerdebildern.

Im Vergleich zu Befindlichkeitsstörungen entsteht bei funktionellen Körperbeschwerden in unterschiedlicher Ausprägung aufgrund der Symptomatik, der Persistenz und der vielfach mit den Beschwerden einhergehenden (psychosozialen) Belastung ein Krankheitswert, der in der Regel auch mit (zum Teil exzessiven) Kontakten zum Gesundheitssystem einhergeht.

Oftmals werden die Patienten, die wiederholte und verschiedene Behandler aufsuchen und mehrfache intensive diagnostische Abklärungen durchführen lassen, auch eher abfällig als „Frequent Flyers“, „Doctor (S)hoppers“ oder „Thick-Folder“-Patienten bezeichnet [2]. 
- Tab. 1 Vom Symptom zur Diagnose - Einordnung von Symptomen in funktionelle Cluster und Diagnosen sowie deren somatische Differenzialdiagnosen.

\begin{tabular}{|c|c|c|c|c|}
\hline Einzel-Symptom (Bsp.) & Kreuzschmerz & Palpitation & Diarrhö & Kraftlosigkeit \\
\hline Häufige Symptom-Cluster & Schmerzen & Kardio-pulmonale Symptome & $\begin{array}{l}\text { Gastrointestinale } \\
\text { Symptome }\end{array}$ & Fatigue \\
\hline $\begin{array}{l}\text { Definierte Diagnose ICD-10 } \\
\text { (Bsp.) }\end{array}$ & $\begin{array}{l}\text { Anhaltende somato- } \\
\text { forme Schmerzstörung }\end{array}$ & $\begin{array}{l}\text { Somatoforme Funktionsstörung } \\
\text { des kardiovaskulären und/oder } \\
\text { respiratorischen Systems }\end{array}$ & Reizdarmsyndrom & Fibromyalgie Syndrom \\
\hline $\begin{array}{l}\text { Differenzialdiagnose soma- } \\
\text { tisch bzw. abwendbar ge- } \\
\text { fährlicher Verlauf (Bsp.) }\end{array}$ & Wirbelkörperfraktur & Vorhofflimmern & $\begin{array}{l}\text { Chronisch entzünd- } \\
\text { liche Darmerkran- } \\
\text { kung }\end{array}$ & $\begin{array}{l}\text { Hämatoonkologische } \\
\text { Grunderkrankung }\end{array}$ \\
\hline
\end{tabular}

\section{FALLBEISPIEL}

Eine 22-jährige Jura Studentin berichtet von einer seit 2 Jahren bestehenden ausgeprägten Müdigkeit und Kraftlosigkeit, welche sie im Alltag sehr einschränke. Weiterhin habe sie Schmerzen „am ganzen Körper“, die in ihrer Lokalisation immer wieder wechseln würden. In ihrer Konzentration sei sie eingeschränkt und sie verspüre ein nicht näher beschreibbares Schwindel- und Benommenheitsgefühl, welches sowohl in Ruhe als auch in Bewegung auftrete. Sie habe große Schlafschwierigkeiten (Ein- und Durchschlafstörungen). Depressive oder andere psychische Symptome berichtet sie nicht. Diverse Abklärungen (cMRT, Lumbalpunktion, diverse Blutentnahmen, EEG, NLG, HNO-Untersuchungen etc.) hätten keinen weiterführenden Befund erbracht.

\section{Symptome}

Klinisch präsentieren sich funktionelle Körperbeschwerden primär als Symptome (z. B. Schmerzen, Fatigue, Palpitationen), deren Einordnung in vielen Fällen ohne Krankheit bleibt (auch oftmals international als „Persistant physical Symptoms“ oder „Medically unexplained (physical) Symptoms" beschrieben) (s. - Tab. 1).

\section{Syndrome und Cluster}

Funktionelle Körperbeschwerden können auch in Clustern oder funktionellen somatischen Syndrom-Kategorien (z. B. Reizdarmsyndrom, Fibromyalgie Syndrom) zusammengefasst werden. Häufige Cluster sind „Schmerz“, „kardiopulmonal“, „gastrointestinal“ und „Fatigue“-assoziiert [3] (s. > Tab.1).

\section{Somatoforme Störungen (ICD-10)}

Bei Erfüllung der Kriterien ist auch eine Einordnung der Symptomatik in eine definierte Diagnosekategorie der Somatoformen Störungen (ICD-10 Kapitel F45) möglich (s. T Tab. 2). Hierbei ist in der aktuellen Klassifikation ICD-10 der Ausschluss einer die Symptomatik erklärenden organischen Erkrankung obligat. Diese geforderte Ausschlussdiagnostik hat oftmals zur Folge, dass Patienten in eine Spirale der Überdiagnostik geraten, mit entsprechenden Folgen (,iatrogene Chronifizierung“, Vermittlung der Nachrangigkeit psychosozialer Aspekte, Verstärkung dualistischer und hierarchischer Erklärungsmodelle, Nebenwirkungen v.a. invasiver Diagnostik etc.).

\section{Die neuen „Somatischen Belastungs- störungen“ gemäß DSM-5 und ICD-11}

Im DSM-5 (Somatic Symptom Disorder) als auch voraussichtlich im ICD-11 (Bodily Distress Disorder) ist der Ausschluss einer somatischen Erkrankung kein obligates Kriterium für funktionelle Körperbeschwerden mehr (siehe Kasten „Bodily Distress Disorder). Anstatt eben dieser (bisher bei somatoformen Diagnosen obligaten) Forderung nach einer organischen Unerklärbarkeit der Beschwerden, sind bei diesen neuen Diagnosen vielmehr psychobehaviorale Kriterien für die Diagnosestellung von Bedeutung:

- Fokussierung auf die Beschwerden,

- häufige Kontakte mit dem Gesundheitswesen,

- keine anhaltende Aus- bzw. Ablenkung von den Beschwerden durch klinische Diagnostik und/oder durch adäquate Beruhigung etc.

\section{Merke}

In der neuen Klassifikation wird die vorliegende Belastung durch die Beschwerden mit den damit einhergehenden Folgen zum wichtigen Diagnose-Kriterium (daher auch der Name „Somatische Belastungsstörung“).

Die Konsequenz dieser neuen Diagnosekategorien ist, dass belastete Patienten frühzeitig eine adäquate Behandlung erfahren können, ohne dass eine den Patienten (und z. T. auch den Behandler) weiter dysfunktional fixierende (und auch potenziell für den Patienten schädigende) Ausschlussdiagnostik vorgenommen werden muss. 
- Tab.2 Auswahl klinisch häufiger somatoformer Störungen ICD-10 [5].

\begin{tabular}{|c|c|}
\hline ICD-10-Klassifikation & Beschreibung \\
\hline Somatisierungsstörung F45.0 & $\begin{array}{l}\text { - Multiple Beschwerden jedweder Art oder Lokalisation ( } \geq 6 \text { aus } \geq 2 \text { Bereichen), wiederholt auftretend und } \\
\text { häufig wechselnd. } \\
\text { - Seit mind. } 2 \text { Jahren: Meist lange, komplizierte „Patientenkarrieren“. } \\
\text { - Verlauf: chronisch und fluktuierend. } \\
\text { - Häufig Störungen des sozialen, interpersonalen, familiären Verhaltens. }\end{array}$ \\
\hline $\begin{array}{l}\text { Undifferenzierte Somatisie- } \\
\text { rungsstörung (F45.1) }\end{array}$ & $\begin{array}{l}\text { - Die körperlichen Beschwerden sind zahlreich, unterschiedlich und hartnäckig, das vollständige und typische } \\
\text { klinische Bild einer Somatisierungsstörung ist aber nicht erfüllt. }\end{array}$ \\
\hline $\begin{array}{l}\text { Sonstige/nicht näher bezeich- } \\
\text { nete somatoforme Störungen } \\
(\text { F45.8/9) }\end{array}$ & $\begin{array}{l}\text { - Alle anderen Störungen der Wahrnehmung, der Körperfunktion und des Krankheitsverhaltens, die nicht durch } \\
\text { das vegetative Nervensystem vermittelt werden, die auf spezifische Teile oder Systeme des Körpers begrenzt } \\
\text { sind und mit belastenden Ereignissen oder Problemen eng in Verbindung stehen, können hier klassifiziert } \\
\text { werden. }\end{array}$ \\
\hline $\begin{array}{l}\text { Anhaltende somatoforme } \\
\text { Schmerzstörung (F45.40) }\end{array}$ & $\begin{array}{l}\text { - Andauernder (mehr als } 6 \text { Monate), schwerer und quälender Schmerz, der durch einen physiologischen Prozess } \\
\text { oder eine körperliche Störung nicht hinreichend erklärt werden kann. } \\
\text { - Der Schmerz wird nicht absichtlich erzeugt oder vorgetäuscht. } \\
\text { - Auftreten in Verbindung mit emotionalen Konflikten oder psychosozialen Belastungen, denen die Hauptrolle } \\
\text { für Beginn, Schweregrad, Exazerbation oder Aufrechterhaltung der Schmerzen zukommt. } \\
\text { - Meist beträchtlich gesteigerte persönliche oder medizinische Hilfe und Unterstützung. }\end{array}$ \\
\hline $\begin{array}{l}\text { Chronische Schmerzstörung } \\
\text { mit somatischen und psy- } \\
\text { chischen Faktoren (F45.41) }\end{array}$ & $\begin{array}{l}\text { - Andauernde ( } \geq 6 \text { Monate) Schmerzen in einer oder mehreren anatomischen Regionen, die ihren Ausgangs- } \\
\text { punkt in einem physiologischen Prozess oder einer körperlichen Störung haben. } \\
\text { - Psychischen Faktoren wird eine wichtige Rolle für Schweregrad, Exazerbation oder Aufrechterhaltung der } \\
\text { Schmerzen beigemessen, jedoch nicht die ursächliche Rolle für deren Beginn. } \\
\text { - Der Schmerz verursacht in klinisch bedeutsamer Weise Leiden und Beeinträchtigungen in sozialen, beruflichen } \\
\text { oder anderen wichtigen Funktionsbereichen. }\end{array}$ \\
\hline
\end{tabular}

\section{PRAXISTIPP}

Funktionelle Körperbeschwerden präsentieren sich unterschiedlich und können als (Einzel)Symptome, in Symptomclustern bzw. als definierte funktionelle Syndromkategorien oder im Rahmen von Diagnosekategorien der somatoformen Störungen bzw. somatischen Belastungsstörungen auftreten.

\section{Einordnung der Symptome: Sowohl-als-auch-Vorgehen}

Die Einordnung „funktionell“ ist bei den ersten Präsentationen oftmals eine Herausforderung; sie sollte jedoch von Anfang an - gleichwertig wie potenziell abwendbar gefährliche Verläufe der Beschwerden - mitbedacht werden (Sowohl-als-auch-Vorgehen).

Bei funktionellen Körperbeschwerden ist überwiegend nicht die Struktur, sondern die Funktion von Organen beeinträchtigt. Gleichzeitig kann oft auch eine gewisse „Funktionalität“ der Beschwerden identifiziert werden (z. B. Entlastung von familiären oder arbeitsbezogenen Konflikten).

\section{BODILY DISTRESS DISORDER}

\section{Ausblick voraussichtliche Diagnose ICD-11}

- Belastung durch die Körperbeschwerden.

- Starke Fokussierung der Aufmerksamkeit auf die Körperbeschwerden.

- Ggf. häufige Kontakte mit dem Gesundheitswesen.

- Bei Vorliegen einer anderen, die Beschwerden (mit-)verursachenden Erkrankung ist die Fokussierung der Aufmerksamkeit hier deutlich stärker ausgeprägt im Vergleich zu dem zu erwartenden Beschwerdeausmaß und -verlauf.

- Keine anhaltende Aus- bzw. Ablenkung von den Beschwerden durch angebrachte klinische Untersuchungen und Diagnostik sowie adäquate Beruhigung.

- Körperliche Beschwerden sind anhaltend und treten an den meisten Tagen über mehrere Monate hinweg auf.

- Typischerweise sind mehrere Körperbeschwerden vorhanden und diese wechseln über die Zeit.

- In Einzelfällen kann auch nur ein Einzelsymptom vorliegen meist Schmerz oder Fatigue - welches dann mit den anderen Kriterien von „Bodily Distress” einhergeht. 


\section{Häufigkeit, Verlauf und Prognose von Funktionellen Körperbeschwerden}

Die Prävalenz von funktionellen Körperbeschwerden in der Allgemeinbevölkerung wird mit etwa $10 \%$ angegeben [6]; sie kommen somit häufig vor.

Merke

In der ambulanten Versorgung, im nicht selektierten Patientengut, gehören funktionelle Körperbeschwerden (zusammengenommen) zu den häufigsten Beratungsanlässen.

Im hausärztlichen Bereich betragen die Prävalenzen 20-50\%. Ein internationaler Review von Haller et al. zeigte, dass bei 40-49\% aller Hausarztpatienten mehr als mindestens eine medizinisch unerklärte Beschwerde vorlag. Ein Allgemeinarzt mit 40 Patienten am Tag sieht ca. 2 Patienten mit funktionellen Körperbeschwerden pro Stunde.

In spezialisierten Settings, wie beispielsweise rheumatologischen, gynäkologischen oder schmerzmedizinischen Ambulanzen, lagen die Häufigkeiten zwischen 25 und $66 \%[7-10]$.

PRAXISTIPP

Es ist wichtig, den Patienten mit funktionellen Körperbeschwerden zu vermitteln, dass sie mit den Beschwerden nicht allein sind („Universalität des Leidens“).

Der Verlauf funktioneller Körperbeschwerden ist im Hinblick auf Art, Lokalisation, Ausmaß, Schweregrad, Auswirkungen auf Lebensqualität und Leistungsfähigkeit sehr variabel.

Erfüllen Patienten bereits Kriterien für eine „Somatoforme Störung“, eine „(multi)-somatoform Disorder“ oder eine „Bodily Distress Disorder“, so sind bei mindestens $20 \%$, eher $50 \%$ der Patienten, die Beschwerden anhaltend [11-14]. Insgesamt berichten im Verlauf $50-75 \%$ der Patienten über eine Besserung ihrer Beschwerden, bei 10-30\% kommt es jedoch zu einer Verschlechterung [15]. Selbst bei ausgeprägter, belastender Beschwerdesymptomatik mit einhergehenden (psychosozialen) Belastungen ist durch einen adäquaten Umgang mit dem Patienten im Verlauf bei $50-75 \%$ der Patienten eine Verbesserung der Beschwerden zu verzeichnen.
PRAXISTIPP

Die Vermittlung der prognostischen Information, dass eine Beschwerdeverbesserung bei der Mehrheit der Patienten möglich ist, kann für Patienten mit funktionellen Körperbeschwerden eine bedeutende Entlastung darstellen.

Beschwerdeanzahl und dysfunktionale Erwartungen (insbesondere katastrophisierende Interpretationen) sind die wichtigsten Prädiktoren für einen chronischen Verlauf. Ebenso geht ein polysymptomatischer Verlauf mit einer hohen psychiatrischen Komorbidität (v. a. Depression, Angst, Sucht, Posttraumatische Belastungsstörung), einer hohen funktionellen Beeinträchtigung und hohen Inanspruchnahme von Gesundheitsleistungen einher. Disproportional zum fehlenden oder nicht hinreichend erklärbaren körperlichen Befund gehen funktionelle Körperbeschwerden häufig mit Einschränkungen von Leistungsfähigkeit und Lebensqualität sowie hohen direkten und indirekten Gesundheitskosten einher $[2,16]$.

Die Lebenserwartung scheint, mit Ausnahme eines erhöhten Suizidrisikos, normal zu sein (einzelne Studien berichten sogar von einer niedrigeren Mortalität bei diesem oft besonders gut untersuchten Kollektiv) [16].

\section{Ätiologische Modelle}

Die Ätiologie funktioneller Körperbeschwerden ist letztendlich ungeklärt. Man geht von einer multifaktoriellen Genese aus, die aus verschiedenen und unterschiedlich gewichteten biopsychosozialen Faktoren besteht. Didaktisch kann unterschieden werden zwischen:

- prädisponierende Faktoren - belastende Lebensumstände, ungünstige Kindheitserfahrungen, (unterschwellige) körperliche Erkrankungen, (epi-)genetische Faktoren, kulturelle Grundannahmen

- auslösende Faktoren - akute Belastungen und Erkrankungen, Unfälle, u.a.

- aufrechterhaltende Faktoren - vorausgegangene (un)angemessene Behandlungen, Vermeidung und Dekonditionierung, Katastrophisieren, ständiges Überprüfen des Körpers auf Symptome jedweder Art („Body Checking Behaviour“), ungünstige Erfahrungen im Gesundheitswesen u. a.

Dabei sind die einzelnen Faktoren nicht immer eindeutig voneinander zu trennen. 


\section{"Theragnostics“: Diagnostik und Therapie funktioneller Körperbeschwerden}

Eine klare Abgrenzung von Diagnostik- und TherapiePhase des Behandlungsprozesses ist bei funktionellen Körperbeschwerden nicht möglich. Man kann daher von einem „theragnostischen Prozess“ sprechen. So ist der Behandler bei der Diagnostik (vor allem bei der Anamnese und bei der körperlichen Untersuchung) durch seine Haltung (im Ideal-Fall) bereits therapeutisch tätig und kann so den möglichen Verlauf funktioneller Körperbeschwerden positiv beeinflussen.

\section{PRAXISTIPP}

Machen Sie sich bewusst, dass Sie bei der Anamnese und der körperlichen Untersuchung bereits therapeutisch tätig sind, einen wichtigen Beziehungsaufbau pflegen und den Verlauf der Symptomatik damit weitreichend beeinflussen können.

Gleichzeitig ist im Laufe der Therapie auch immer wieder der „diagnostische Blick“ auf die Beschwerdesymptomatik gefragt, um Warnzeichen für körperliche Erkrankungen oder evtl. Folgeschäden vorausgegangener Passivierung oder Fehlbehandlungen frühzeitig zu erkennen (Sowohl-als-auch-Haltung).

Viele Behandler zögern, eine funktionelle oder psychosomatische Diagnose zu vergeben aus Sorge eine organische Erkrankung zu „verpassen“. Dabei ist die Rate an fälschlich als „funktionell“ eingeordneten Beschwerden niedrig, wenngleich nicht vernachlässigbar. In Followup-Untersuchungen musste bei 0,5\% der Patienten die Diagnose „funktioneller Körperbeschwerden“ revidiert werden, weil die Beschwerden durch eine zugrunde liegende somatische Erkrankung besser erklärbar waren (im Rahmen von „diagnostic evaluation studies“, in denen Patienten mit kürzlich diagnostizierten „Funktionellen Körperbeschwerden“ erneut strukturiert untersucht wurden, lag die Rate der revidierten Diagnosen nach erneuter Diagnostik bei 8,8\%) [17].

\section{Merke}

Die Abklärung/Diagnostik und Therapie (bzw. bewusste „Nicht-Diagnostik“ und „Nicht-Therapie“) sollte nach Schweregraden gestuft erfolgen (s. Abb. 1).

Für den Verlauf der Beschwerden ist entscheidend, funktionelle Körperbeschwerden möglichst frühzeitig als solche zu identifizieren und damit einen „Switch“ im Umgang mit dem Patienten vorzunehmen.

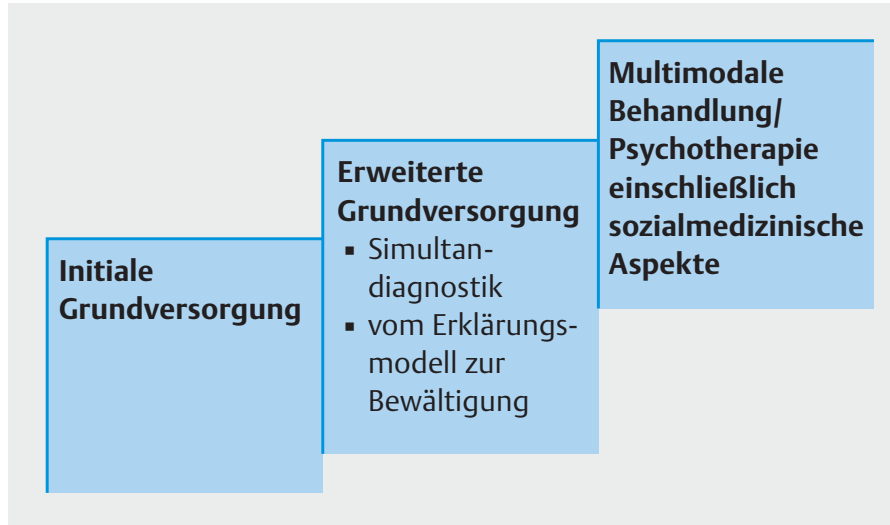

Abb. 1 Stufenmodell der Versorgung von Patienten mit funktionellen Körperbeschwerden.

\section{Den „Hut“ aufbehalten - Stufenmodell nach Schweregraden und Behand- lungsphasen}

Der Behandlungsprozess wird in 3 Behandlungsphasen unterteilt, die im Hinblick auf Versorgungsform und den Schweregrad der Beschwerden aufeinander aufbauen (s. Abb. 1). Die initiale und erweiterte Grundversorgung erfolgt in der Regel durch den Behandler der Primärversorgung (i. d. R. der Hausarzt oder der somatische Facharzt). Aus diesem Grund richtet sich der Hauptteil der Empfehlungen auch an diese Behandler und adressiert nicht primär die Kolleginnen und Kollegen der Psychiatrischen und Psychotherapeutischen Disziplinen - wenngleich jedoch die Empfehlungen auch für die Kollegen dieser Disziplinen von Interesse sein können (und sollten!). Bei der weiteren Behandlung, die (spätestens) in der 3. Behandlungsphase weitere Behandler miteinschließt (multimodale Therapie), behält der Primärbehandler als Koordinator, „GateKeeper" und in seiner Funktion als derjenige, bei dem die Informationen zusammenlaufen, „den Hut auf“.

\footnotetext{
Merke

Der Primärbehandler bleibt auch in der multimodalen Therapie der Koordinator und Hauptbezugstherapeut für den Patienten.
}

Die Empfehlungen der initialen Grundversorgung sind weiterhin in den Behandlungsphasen der erweiterten Grundversorgung und im Rahmen der psychotherapeutischen und multimodalen Behandlung von Bedeutung, werden dann aber durch die Empfehlungen der jeweils nachfolgenden Behandlungsstufe ergänzt. 


\begin{tabular}{|c|c|}
\hline $\begin{array}{l}\text { SCHUTZFAKTOREN } \\
\text { FÜR EINEN } \\
\text { GÜNSTIGEN VERLAUF } \\
\text { („GREEN FLAGS“) }\end{array}$ & $\begin{array}{l}\text { - Funktionale Gedanken und Einstellungen, z. B. positive } \\
\text { Lebenseinstellung, Humor, Selbstbewusstsein } \\
\text { - Aktive Bewältigungsstrategien, z. B. sportliche Betätigung, aber auch } \\
\text { Genuss- und Entspannungsfähigkeit } \\
\text { - Individuelle Ressourcen, z. B. Hobbies, soziales Engagement, } \\
\text { berufliche Pläne } \\
\text { - Keine oder geringe psychosoziale Belastung, z. B. stabile Bindungen, } \\
\text { gute soziale Unterstützung, gute Lebens- und Arbeitsbedingungen } \\
\text { - Keine psychische Komorbidität } \\
\text { - Weitgehend erhaltene Funktionsfähigkeit, z. B. Berufstätigkeit oder } \\
\text { Sozialkontakte } \\
\text { - Tragfähige Arzt-Patient-Beziehung } \\
\text { - Biopsychosozialer Behandlungsansatz mit Vermittlung von Zuversicht } \\
\text { und positiven Bewältigungsstrategien, unter Vermeidung unnötiger } \\
\text { Diagnostik und Therapie }\end{array}$ \\
\hline $\begin{array}{l}\text { INDIKATOREN/ } \\
\text { RISIKOFAKTOREN } \\
\text { FÜR EINEN } \\
\text { SCHWEREREN } \\
\text { VERLAUF } \\
\text { („YELLOW FLAGS“) }\end{array}$ & $\begin{array}{l}\text { - Mehrere Beschwerden (polysymptomatischer Verlauf) } \\
\text { - Häufige bzw. anhaltende Beschwerden (ohne oder nur mit seltenen } \\
\text { beschwerdefreien Intervallen) } \\
\text { - Dysfunktionale Gedanken und Einstellungen z. B. Katastrophisieren, } \\
\text { Hilf-/Hoffnungslosigkeit, Angst-Vermeidungs- Überzeugungen, hohe } \\
\text { gesundheitsbezogene Angst } \\
\text { - Passives, überaktives oder suppressives Verhalten, z. B. ausgeprägtes } \\
\text { Schon- und Vermeidungsverhalten, beharrliche Arbeitsamkeit/ } \\
\text { Durchhalte-Verhalten, hohes Inanspruchnahmeverhalten } \\
\text { - Mäßige bis hohe psychosoziale Belastung, z. B. Distress (negativer } \\
\text { Stress, vor allem berufs-/arbeitsplatzbezogen), Niedergeschlagenheit, } \\
\text { Zukunftsängste, Einsamkeit } \\
\text { - Psychische Komorbidität (v.a. Depressionen, Angststörungen, } \\
\text { Suchterkrankungen, Traumafolgestörungen) } \\
\text { - Deutlich reduzierte Funktionsfähigkeit, z. B. Arbeitsunfähigkeit, } \\
\text { sozialer Rückzug, körperliche Dekonditionierung } \\
\text { - Behandler-Patient-Beziehung wird von beiden Seiten als „,schwierig“ } \\
\text { erlebt } \\
\text { - „latrogene Somatisierung“ bzw. „Chronifizierung“ durch } \\
\text { Nocebobotschaften, Passivierung, unnötige Diagnostik und Therapie }\end{array}$ \\
\hline $\begin{array}{l}\text { WARNHINWEISE } \\
\text { („RED FLAGS“) }\end{array}$ & $\begin{array}{l}\text { - Selbstgefährdung bis hin zu Suizidalität (z. B. massive } \\
\text { Mangelernährung/Untergewicht, schwere körperliche Folgeschäden } \\
\text { von Schonung wie Kontrakturen, Selbstmordgedanken und -pläne) } \\
\text { - Gefährdung durch Andere, z. B. durch Ärzte, meist durch fehlende } \\
\text { oder ungeeignete Behandlungen (z. B. Vorenthaltung notwendiger } \\
\text { Therapien körperlicher Begleiterkrankungen, Mangelsyndrome durch } \\
\text { Ausleittherapien, hochriskante invasive Therapien wie nicht indizierte } \\
\text { Operationen) } \\
\text { - Besonders schwere psychische Komorbidität (z. B. völliger Rückzug } \\
\text { bei Angsterkrankungen oder Depressionen) } \\
\text { - Warnsignale bekannter körperlicher Erkrankungen (z. B. Blut im Stuhl } \\
\text { bei Magen-Darm-Beschwerden, B-Symptomatik bei Erschöpfung, } \\
\text { Probleme mit Stuhlgang oder Wasserlassen bei Rückenschmerzen) }\end{array}$ \\
\hline
\end{tabular}

- Abb.2 Schutzfaktoren, Risikofaktoren für schweren Verlauf und Warnhinweise (Deutsche Gesellschaft für Psychosomatische Medizin und Ärztliche Psychotherapie e. V. (DGPM), Deutsches Kollegium für Psychosomatische Medizin (DKPM), u. a. S3-Leitlinie „Funktionelle Körperbeschwerden“ In: Arbeitsgemeinschaft der Wissenschaftlichen Medizinischen Fachgesellschaften e.V. (AWMF) e. V.; 2018 [5]). [rerif] 
Die Einteilung der Beschwerdesymptomatik nach Schweregraden orientiert sich an Schutzfaktoren und Risikofaktoren für einen schweren Verlauf. So unterscheidet man hier zwischen sogenannten

- „green flags“, also Schutzfaktoren für einen günstigen Verlauf,

- „yellow flags“, also Indikatoren bzw. Risikofaktoren für einen schweren Verlauf und

- „red flags“ in Form von Warnhinweisen auf abwendbar gefährliche Erkrankungen, die ein unmittelbares Handeln voraussetzen (\$ Abb. 2) [2].

\section{Initiale Grundversorgung}

Zu den Pfeilern der initialen Grundversorgung gehören als „Anfangsinvestition“ zunächst Wachsamkeit, Zurückhaltung und Empathie (s. > Abb. 3).

\section{Bemerken und Erkennen}

Da funktionelle Körperbeschwerden häufig sind und sich vielgestaltig präsentieren, sollte frühzeitig im Behandlungsverlauf die Möglichkeit erwogen werden, dass die von den Patienten vorgetragenen Beschwerden funktioneller Natur sind. So kann der weitere Verlauf der Beschwerden positiv beeinflusst werden. Hierbei sollte die Haltung von Wachsamkeit, Zurückhaltung und Empathie geprägt sein.

\section{Sorgfältig befragen und untersuchen}

Die Patienten sollten in der Anamnese nach ihren Hauptbeschwerden, aber auch nach weiteren Beschwerden oder Problemen gefragt werden. Um einen Eindruck von den Beeinträchtigungen durch die Beschwerden im Alltag und einer eventuellen psychischen Belastung zu bekommen, sollte auch danach gefragt werden:

- Wie es den Patienten mit den Beschwerden geht?

- Wie sich die Beschwerden auswirken (in der Lebensführung, im sozialen oder beruflichen Umfeld)?

- Wie sie damit umgehen?

\section{Merke}

Einen wichtigen Stellenwert haben bei der initialen Grundversorgung die Anamnese und klinische Untersuchung.

Eine anschließende, angekündigte sorgfältige, allgemeine körperliche Untersuchung soll ggf. Hinweise auf weitere Beschwerden, Befunde und Einschränkungen erfassen. Im Rahmen des Kontaktes ist sowohl im Gespräch als auch während der körperlichen Untersuchung auf das Verhalten der Patienten zu achten (z. B. ängstliche Vermeidung von Bewegungen, dramatisierender Beschwerdeausdruck). (Vor-)Befunde sollten entsprechend mit den Patienten besprochen und die weitere Diagnostik nach Schweregraden gestuft, systematisch, aber zurückhaltend durchgeführt werden.

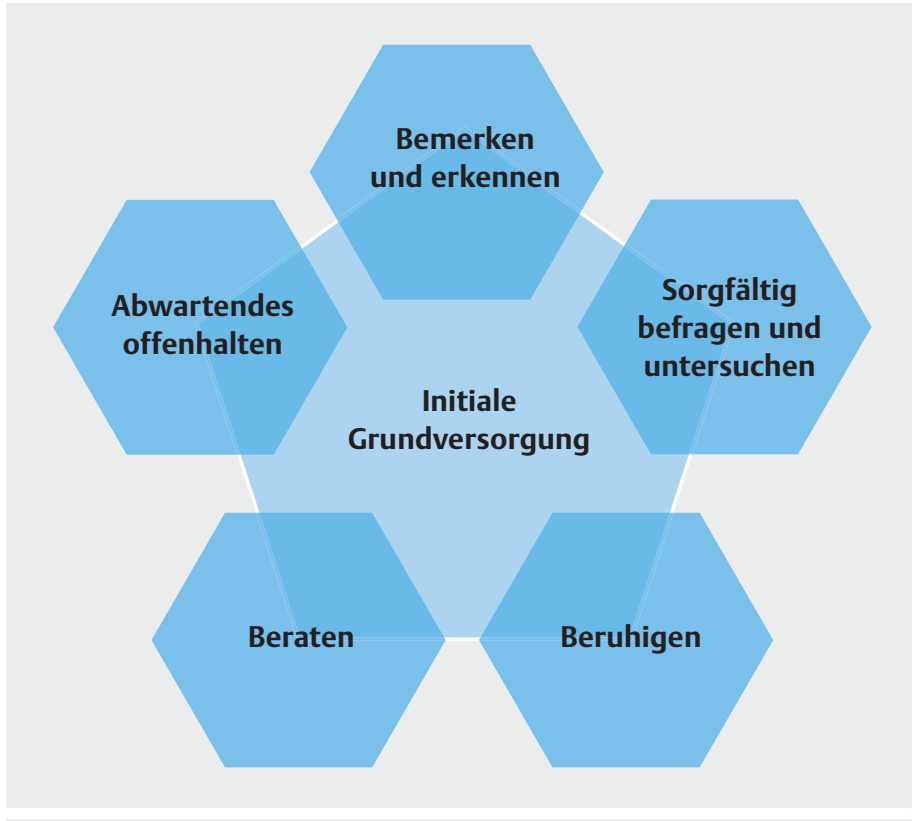

- Abb.3 Empfehlungen der initialen Grundversorgung.

Auf der Basis aller erhobenen Informationen und Befunde sollte auf eventuell vorliegende Hinweise für abwendbar gefährliche Verläufe oder auf Risikofaktoren für einen chronischen Verlauf geachtet werden (s.

- Abb. 4).

\section{FALLBEISPIEL}

Ein 32-jähriger Flüchtling aus Syrien kommt in die Praxis und berichtet über unspezifische Beschwerden (Rückenschmerzen, Druck im Kopf und hinter den Augen, „Kribbelgefühl“ in allen 4 Extremitäten, Schmerzen im Unterbauch bei voller Blase).

In der körperlichen Untersuchung zuckt der Patient bei Berührungen mehrfach zusammen und bei der Inspektion zeigen sich diverse Narben an den Extremitäten und am Rücken. Eine Tätowierung am linken Knöchel zeigt ein Auge mit einer Träne im Augenlid.

Die behandelnde ärztliche Kollegin thematisiert in den Folgegesprächen empathisch ihre Beobachtungen. Der Patient öffnet sich bei ihr dann erstmals einem Behandler gegenüber mit seiner „Vorgeschichte“ und berichtet über traumatisierende körperliche Misshandlungen auf seiner Flucht. Sie entwerfen gemeinsam ein biopsychosoziales Beschwerdemodell und den weiteren Behandlungsplan. 


\begin{tabular}{|l|l|}
\hline Simultandiagnostik & $\begin{array}{l}\text { Vom Erklärungsmodell } \\
\text { zur Bewältigung }\end{array}$ \\
\hline - Entschleunigen und & - Erklären und benennen \\
$\begin{array}{l}\text { Behandlungssetting } \\
\text { anpassen }\end{array}$ & - An Erwartungen und \\
- Aufmerksam zuhören & Zielen arbeiten \\
und nachfragen & - Beschwerden und \\
- Wohlüberlegt unter- & Symptome lindern \\
suchen und anordnen & - Selbstfürsorge und \\
- Untersuchungen & Selbstwirksamkeit \\
besprechen & stärken \\
- Krankheitswert und & - Zur körperlichen \\
diagnostische Zuord- & Aktivität ermutigen \\
nung(en) klären & - Zusammenarbeiten und \\
& Rat einholen \\
\hline &
\end{tabular}

- Abb.4 Empfehlungen der erweiterten Grundversorgung - unterteilt in „Simultandiagnostik“ und „Vom Erklärungsmodell zur Bewältigung“.

\section{Beruhigen}

Falls aus Anamnese und Untersuchungsbefund keine Hinweise für abwendbar gefährliche Verläufe bekannter körperlicher oder psychischer Erkrankungen gefunden wurden, sollte dies den Patienten mitgeteilt werden, um sie zu beruhigen. Hierbei sollte das Gefühl von Ernstgenommen-Werden, Sorgfalt und Sicherheit sowie insbesondere die Glaubwürdigkeit der Beschwerden vermittelt werden, ohne dabei notwendigerweise bereits auf eine Diagnose zurückzugreifen, aber auch ohne die Beschwerden zu verharmlosen oder zu negieren.

\section{Beraten}

Schon frühzeitig im Behandlungsverlauf sind allgemeine Maßnahmen zu erfragen, welche geeignet sind, die Symptome günstig zu beeinflussen (im Sinne eines gesunden, körperlich aktiven Lebensstils), bzw. sollte den Patienten zunächst zu solchen gesundheitsfördernden Maßnahmen geraten werden, mit denen diese ggf. früher in vergleichbaren Situationen positive Erfahrungen gemacht haben („Patientenpräferenzen“).

\section{PRAXISTIPP}

Maßnahmen, welche geeignet sind, die Symptome günstig zu beeinflussen, sind z. B.:

- „Hausmittel“ wie einfache Rituale und Traditionen, die zu Entspannung, Wohlbefinden und Beschwerdelinderung führen

- körperliche Aktivierung durch genussvolle Alltagsbewegung und einfache Alltagssportarten

- soziale Aktivierung und Austausch im „Kleinen“ (Familie, enge Freunde) und „Großen“ (Bekanntenkreis, Gruppenaktivitäten etc.)

\section{Abwartendes Offenhalten}

Im Sinne der Strategie des abwartenden Offenhaltens sollte man den Patienten bei Bedarf ein Wiedervorstellungstermin in 2-4 Wochen anbieten. Ausdrücklich ist zu betonen, dass sich die Beschwerden wahrscheinlich bessern und auch kein Grund zur Sorge besteht, falls sie persistieren.

\section{PRAXISTIPP}

Eine empathische, wachsame und von Zurückhaltung geprägte Haltung im Umgang mit dem Patienten wirkt sich positiv auf die beiderseitige Behandlungszufriedenheit und den Behandlungsverlauf aus (z. B. Zeitersparnis bei Folgekontakten, Schaffung von Ressourcen, Adhärenz, Lebensqualität).

\section{Erweiterte Grundversorgung \\ Simultandiagnostik}

Die erweiterte Grundversorgung ist bei persistierend beeinträchtigenden Beschwerden angezeigt und bezieht sich auf den bewussten weiteren Umgang, vor allem im Hinblick auf die Diagnostik im Verlauf (Simultandiagnostik) und die Entwicklung eines gemeinsamen Erklärungsmodells ( $\triangleright$ Abb. 4).

\section{Entschleunigen und Behandlungssetting anpassen}

Der weitere Umgang mit Patienten mit funktionellen Körperbeschwerden sollte in einem klaren Setting mit festen, regelmäßigen, beschwerdeunabhängigen Terminen in ruhiger Atmosphäre etabliert werden.

Um sich und den Patienten mehr Zeit zu widmen (z. B. Zeit zum Nachdenken, alte Befunde anzusehen, mit Vor- oder Mitbehandlern zu sprechen, evtl. einen Hausbesuch oder eine Fremdanamnese zu machen), sollte die Praxisorganisation und Abrechnung auf diese Aspekte hin überprüft werden. So kann zusammen mit 
den medizinischen Fachangestellten bzw. im Team ein bewusster Umgang mit diesen Patienten entwickelt werden (z. B. klares Terminmanagement, interne Kommunikation).

\section{Aufmerksam zuhören und nachfragen}

Die ersten Gesprächsphasen sollten als „Bühne des Patienten“ betrachtet werden. Wichtig ist es hier, nicht zu früh zu unterbrechen, zunächst offene, neutrale Fragen zu stellen (z. B. „Wie fühlen Sie sich (mit ihren Beschwerden)? Wie kommen Sie damit zurecht?") und vor allem aktiv und aufmerksam zuzuhören.

\section{Merke}

Aktives Zuhören wird, neben dem aufmerksamen Zuhören, unterstützt durch authentische und empathische Mimik und Gestik (z. B. Nicken, kurze Bestätigungslaute, Blickkontakt).

Bei der Beschwerdepräsentation sollte auch auf Stimmung, Körpersprache, Zwischentöne, Betontes, Verharmlostes, Ursachenannahmen sowie auf weitere Beschwerden und Belastungen geachtet werden, auch wenn sich diese außerhalb des eigenen Fachgebiets befinden. Hierbei sollte auch die Zeit während der körperlichen Untersuchungen genutzt werden (s. - Tab.3).

Aber auch das eigene Empfinden, Verhalten sowie die Beweggründe in der Arzt-Patient-Interaktion (z. B. Hilflosigkeit, übereifriges Anordnen, wiederholte Arbeitsunfähigkeitsbescheinigungen) sollten beobachtet werden.

Es ist wichtig, den Betroffenen zu zeigen, dass man ihren Leidensdruck bemerkt und die Beschwerden ernst nimmt, z. B. durch eine empathische Mimik und Gestik.

Im Rahmen der erweiterten Anamnese sollten im Verlauf des Gesprächs (auch bereits im Hinblick auf mögliche therapeutische Anknüpfungspunkte) folgende Aspekte durch gezieltes Nachfragen Beachtung finden:

- Fokus auf Hauptbeschwerde(n)

- körperliche und psychische Komorbiditäten und Differenzialdiagnosen

- subjektives Erklärungsmodell und Bedeutungszuschreibungen

- frühere und weitere Beschwerden, Begleiterkrankungen und Behandlungen

- problematische Denk- und Verhaltensweisen

- Ressourcen

- psychosozialer Beschwerdekontext einschließlich besonderer psychosozialer Herausforderungen und Belastungen (s. > Abb. 5)
- Tab. 3 Leitfaden für Aspekte der nonverbalen Kommunikation zwischen Patient und Behandler (EMPATHY-Schema nach Riess und Kraft-Todd [18]; modifiziert nach [5]).

\begin{tabular}{|l|l|}
\hline $\mathbf{E}$ (ye contact) & Wie ist der Blickkontakt des Patienten? \\
\hline $\begin{array}{l}\mathbf{M} \text { (uscles of facial } \\
\text { expression) }\end{array}$ & Welche Mimik zeigt der Patient? \\
\hline $\mathbf{P}$ (osture) & Wie ist die Haltung des Patienten? \\
\hline $\mathbf{A}$ (ffect) & Welchen Affekt zeigt der Patient? \\
\hline $\mathbf{T}$ (one of voice) & Welchen Tonfall hat der Patient? \\
\hline $\begin{array}{l}\mathbf{H} \text { (“What am I hearing } \\
\text { and what would be } \\
\text { healing for this patient”) }\end{array}$ & $\begin{array}{l}\text { Was höre ich genau („zwischen den Zeilen“) } \\
\text { und wo deutet der Patient etwas an, das ggf. } \\
\text { therapeutisch nutzbar ist? }\end{array}$ \\
\hline \begin{tabular}{l} 
Y(our reaction) \\
\hline
\end{tabular} & \begin{tabular}{l} 
Wie reagiere ich auf den Patienten? \\
\hline
\end{tabular} \\
\hline
\end{tabular}

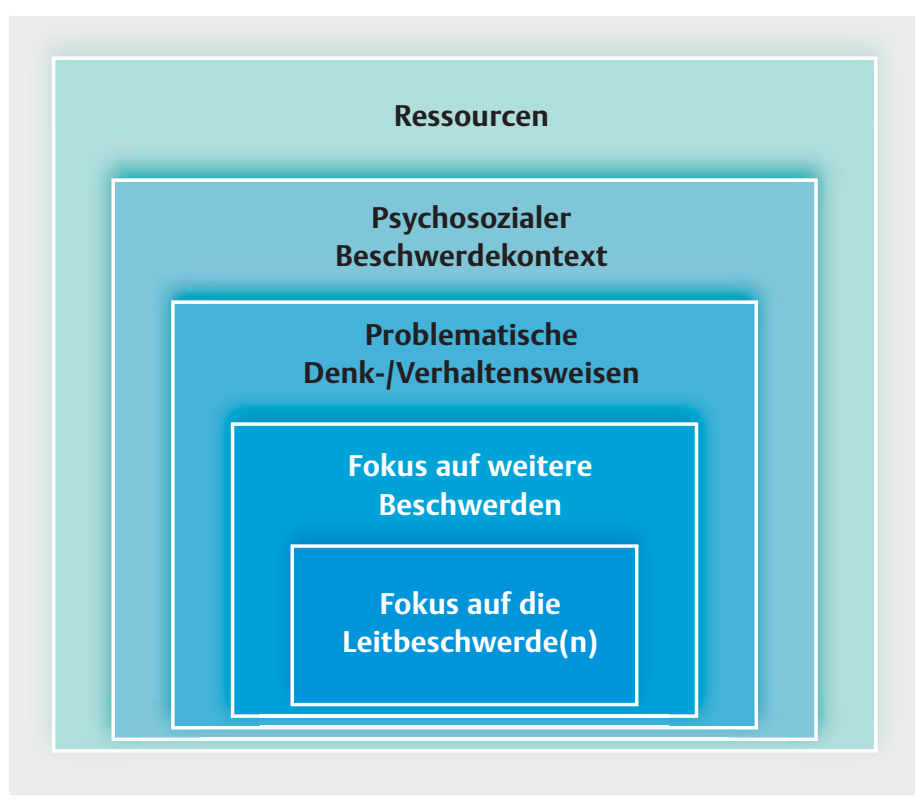

Abb.5 Aspekte der erweiterten Anamnese.

Es ist weiterhin wichtig, sich (und ggf. dem Team) individuelle Besonderheiten und Therapiebedürfnisse aufgrund von Persönlichkeit, Geschlecht, Alter oder Herkunft/Kultur der Patienten bewusst zu machen.

Auch kürzere Gesprächszeiten sollten effizient genutzt werden, indem bewährte Gesprächstechniken einsetzt werden. Beispielsweise können psychosoziale Kontextfaktoren beiläufig und einfühlsam ins Gespräch eingebracht werden und dabei kann man immer wieder auf die Körperbeschwerden zurückkommen (,tangentiale Gesprächsführung“). 
PRAXISTIPP

Beispiel für tangentiale Gesprächsführung: „Letztes Mal erwähnten Sie Stress in der Ehe. Und wie wirkt sich dieser wiederum auf ihre Bauchschmerzen aus?"

\section{Wohlüberlegt untersuchen und anordnen}

Bei der weiteren apparativen und laborchemischen Diagnostik sollte auch bei der erweiterten Grundversorgung systematisch (im Hinblick auf Indikation, Nutzen und Risiken wohlüberlegt und gezielt), gestuft und nicht redundant vorgegangen werden.

\section{Merke}

Ziel ist nicht, für alle Beschwerden eindeutige Ursachen zu benennen (das kann auch die moderne Medizin nicht immer), sondern abwendbar gefährliche Verläufe (z. B. Karzinom, Suizidalität) auszuschließen und Handlungsbedarf zu erkennen (s. - Abb. 2).

Klinisch-körperliche Untersuchungen sollten ggf. in regelmäßigen Abständen wiederholt werden, auch um Warnzeichen für körperliche Erkrankungen oder evtl. Folgeschäden vorausgegangener Passivierung oder Fehlbehandlungen zu erkennen.

Wiederholte, vor allem invasive technische Zusatzuntersuchungen sollten vermieden werden, wenn sie hauptsächlich dazu dienen sollen, den Patienten oder auch den Behandler selbst zu beruhigen.

\section{Untersuchungen besprechen}

Sofern weitere Untersuchungen notwendig sind, sollten diese beruhigend angekündigt werden. Wenn angebracht, sollte schon im Vorfeld auf die hohe Wahrscheinlichkeit eines altersentsprechenden Normalbefundes hingewiesen werden. Wichtig ist es auch, zu erklären, warum bestimmte Untersuchungen nicht notwendig sind.

Frühere Befunde, Zufalls- oder Bagatellbefunde ohne weitere diagnostische oder therapeutische Bedeutung sollten normalisierend eingeordnet werden.

\section{PRAXISTIPP}

Bei mehreren Untersuchungen, die sich über längere Zeit erstrecken, können zwischenzeitliche „Bilanzierungsgespräche“ sinnvoll sein, die die bisherigen Untersuchungen zusammenfassen und bewerten.
Erhaltene Befunde sollten in laiengerechter Sprache besprochen werden. Dies umfasst Formulierungen, die die Patienten beruhigen, informieren und motivieren, anstatt zu riskieren, sie zu verwirren und zu ängstigen. Durch Rückfragen ist sicherzustellen, dass die Informationen korrekt verstanden wurden.

Sowohl bei der Ankündigung als auch bei der Besprechung von Untersuchungen kann man geeignetes Informationsmaterial verwenden (z. B. eine Darmspiegelung in Bildern) und in einfacher Sprache, ggf. auch in einer Fremdsprache erklären.

\section{Krankheitswert und diagnostische Zuordnung(en) klären}

Wichtige Befunde sollten - auch zur Verlaufsbeobachtung für sich selbst und andere Behandler - für ein möglichst klares Bild relevanter Problembereiche (einschließlich psychosozialer Kontextfaktoren) dokumentiert werden. Dabei ist insbesondere auf (neue) Hinweise für abwendbar gefährliche Verläufe oder ein Chronifizierungsrisiko zu achten und daraus eventueller Handlungsbedarf abzuleiten (s. - Abb. 2).

Merke

Aus günstigen Prognosefaktoren lassen sich geeignete therapeutische Anknüpfungspunkte ableiten (s. Abb. 2).

Das eigene Erklärungsmodell für die Beschwerden, z. B. in Form einer Verdachtsdiagnose, sollte geprüft werden und dabei sollte beobachtet werden, wo selbst noch diagnostische Bedenken oder Ängste bestehen, und wie begründet diese sind; dabei sollte an die hohe Wahrscheinlichkeit funktioneller Beschwerden und an die Möglichkeit des abwartenden Offenhaltens gedacht werden, ohne dass sofort Gefahr droht. Diagnostisch ist zu prüfen, inwiefern die Kriterien einer spezifischen funktionellen oder somatoformen Störung erfüllt sind und Krankheitswert und Handlungsbedarf besteht (siehe $>$ Tab. 2). Hierbei sollte auf mögliche körperliche und psychische Komorbiditäten geachtet werden und ggf. sollten mehrere Diagnosen vergeben werden.

\section{PRAXISTIPP}

Falls keine eindeutige Diagnose besteht, kann man die Symptom („R“)- oder Inanspruchnahme („Z“)-Verschlüsselung (z. B. Kopfschmerzen, Übelkeit, psychosoziale Umstände) wählen, anstatt Verlegenheitsdiagnosen zu vergeben. 


\section{Vom Erklärungsmodell zur Bewältigung}

Die weitere Therapie besteht im Rahmen der erweiterten Grundversorgung im Wesentlichen aus der Vermittlung eines nachvollziehbaren, für den Patienten akzeptablen Erklärungsmodells und der Stärkung von Selbstwirksamkeit.

\section{Erklären und benennen}

Im Rahmen der erweiterten Grundversorgung sollte der Patient darauf hingewiesen werden, dass Beschwerden auch ohne körperliche Erkrankungen häufig sind, sie also mit ihren Erfahrungen nicht alleine sind. Psychophysiologische Zusammenhänge, z. B. mithilfe von „Teufelskreismodellen“ („)e mehr Schmerzen, desto weniger Bewegung - je weniger Bewegung, desto mehr Schmerzen“), anatomischen Abbildungen und modernen wissenschaftlichen Erkenntnissen sollten dem Patienten vermittelt werden.

\section{PRAXISTIPP}

Erklären Sie dem Patienten die Beschwerden mit verständlichen Formulierungen als physiologischer Ausdruck (z. B. Zittern, Schwitzen, Herzklopfen) einer Belastungssituation (Anspannung, Stress, Angst, Reizzustand, „aus dem Gleichgewicht geraten“).

Gemeinsam mit dem Patienten sollten ganz persönliche, für ihn gut nachvollziehbare, vorwurfsfreie, multifaktorielle Erklärungsmodelle erarbeitet werden („biopsychosozial“, „sowohl-als-auch“). Diese sollten an die bisherigen Annahmen anknüpfen und gleichzeitig mögliche Lösungen aufzeigen, vor allem aber Veränderungsmöglichkeiten durch die Patienten selbst (z.B. Abbau von Vermeidungs-, Schon- oder Überforderungsverhalten, Lösung von Arbeitsplatzkonflikten) (s. Kasten).

Belastende Kontextfaktoren ebenso wie körperliche (Vor-) Erkrankungen oder Befunde sollten als „Bedingungen“, „Auslöser“, „Verstärker“ bzw. als „zusätzliche Baustellen“ - aber nicht als „Ursachen“ bewertet werden.

\section{Cave}

Monokausale, einseitig psychosoziale oder einseitig somatische Ursachenzuschreibungen sollten vermieden werden.
KRITERIEN FÜR EIN GUTES ERKLÄRUNGSMODELL [19]

- Es ist für Behandler und Patient plausibel.

- Es impliziert keine Schwäche oder Schuld des Patienten.

- Es fördert therapeutische Partnerschaft bzw. Handlungen.

- Es beinhaltet eine deskriptive Bezeichnung, aber nicht zwingend eine spezifische Diagnose.

- Es beschreibt Kausalitäten, z. B. in Form aufrechterhaltender Faktoren, aber nicht notwendigerweise Ursachen.

- Es entsteht aus einem Dialog zwischen Behandler und Patient.

Wenn Diagnosen (einschl. Komorbiditäten) bestehen, sollten diese angemessen erläutert werden. Dabei sind auch Möglichkeiten zur psychischen Entlastung des Patienten durch die Mitteilung der Diagnose und sich daraus ergebender (Be-)Handlungsmöglichkeiten zu nutzen. Funktionelle und somatoforme Diagnosen sollten hier von bekannten bzw. von den Patienten befürchteten anderen Erkrankungen abgegrenzt werden und deren beschreibender Charakter, die normale Lebenserwartung sowie weitere, bewährte Informations-, Therapie- und Selbsthilfemöglichkeiten sollten dem Patienten erklärt werden (s. > Tab. 2).

\section{An Erwartungen und Zielen arbeiten}

Vorbestehende Annahmen und Erwartungen von Patienten (teils auch von Angehörigen) bestimmen das Beschwerdeerleben, ihren Verlauf, das Krankheitsverhalten und den Therapieerfolg. Deshalb sollte man bestehende Annahmen und Erwartungen, aber auch Zweifel und Widersprüche besprechen und erklären und den Patient dazu ermuntern, diese zu überprüfen und neue Erfahrungen zu machen.

Unrealistische Erwartungen sollten relativiert und dem Patienten erklärt werden, dass man auch mit Beschwerden ein gutes Leben haben kann.

\section{Merke}

Das Finden einer eindeutigen „Ursache“, ein schnelles „Wegmachen“ oder „Loswerden“ der Beschwerden oder andere Heilsversprechen sind nicht zielführend.

Gemeinsam sollten mehrere konkrete, möglichst kleinschrittige Zwischenziele im Hinblick auf mehr Selbstwirksamkeit, verbessertes Körpererleben, körperliche und soziale Aktivierung, Regeneration und Entspannung erarbeitet werden.

Weiterhin sollten Behandler und Patient ebenfalls gemeinsam übergeordnete Werte, Motivationen und Therapieziele („Leuchtturm“, „Vision“) erarbeiten, z. B. in Bezug auf eine Vergrößerung des Bewegungsradius, einen Lebenstraum, gesellschaftliches Engagement, 
den Erhalt der Partnerschaft oder der Arbeits- und Erwerbsfähigkeit. Dabei sollte der Therapeut auf Patientenpräferenzen, aber auch mögliche widersprüchliche Ziele (z. B. zwischen Gesundungs- und Rentenwunsch) sowie innere (z.B. Ängste, Konfliktvermeidung) und äußere Behandlungshindernisse (z. B. Dagegen-Arbeiten des Partners, laufende Schmerzensgeldverhandlungen) achten.

Merke

Der Patient sollte bei der Umsetzung seiner Ziele begleitet, motiviert und für schon erreichte Fortschritte gelobt werden.

Erwartungen an die Rollen von Arzt und Patient in der weiteren Behandlung sollten abgestimmt werden. Angestrebt werden sollte eine partizipative Entscheidungsfindung mit einer partnerschaftlichen Zusammenarbeit.

\section{Beschwerden und Symptome lindern}

Der in der Regel vorübergehende und begleitende Charakter von passiven, beschwerdelindernden Maßnahmen sollte im Vergleich zu nachhaltigeren aktiven Selbstwirksamkeitsstrategien erklärt werden und hierbei Vor- und Nachteile dieser Maßnahmen mit dem Patienten abgewogen werden. Der Patient sollte hinsichtlich passiver symptomatischer Maßnahmen zur Beschwerdelinderung beraten werden, z. B.:

- Analgetika

- Psychopharmaka

- physikalische und physiotherapeutische Anwendungen

- passive komplementärmedizinische Begleittherapien wie z. B. Akupunktur

Für Details wird auf die Evidenztabellen mit der Angabe von Evidenzlevels zu einzelnen Therapiemaßnahmen bei konkreten Störungsbildern in der Langfassung der Leitlinie verwiesen (https://www.awmf.org/leitlinien/ detail/II/051-001.html) [5].

\section{Selbstfürsorge und Selbstwirksamkeit stärken}

Wichtig ist die Vermittlung von aktiven, nachhaltigen Bewältigungsstrategien.

Dem Patienten sollte zur (Wieder-)Aufnahme sozialer und körperlicher Aktivitäten und zur (Re-)Exposition bezüglich aktuell vermiedener Aktivitäten geraten werden.

\section{PRAXISTIPP}

Ermutigen Sie Patienten zur (Wieder-)Aufnahme sozialer und körperlicher Aktivitäten. Fragen Sie den Patienten, welche Aktivitäten er früher gerne gemacht hat und motivieren sie ihn, solche wiederzuentdecken.

\section{FALLBEISPIEL}

Ein 56-jähriger Patient hat sich wegen diverser körperlicher Beschwerden (Palpitationen, Übelkeit, Oberbauchschmerzen, mehrfache weiche Stuhlgänge am Tag, Kopfschmerzen) ohne hinreichende organische Erklärung im letzten Jahr immer weiter sozial zurückgezogen. Er ist seit über 6 Monaten krankgeschrieben und verlässt kaum noch das Haus. Der Hausarzt begleitet den Patienten. Im Rahmen von regelmäßigen Gesprächen ist es gemeinsam gelungen (shared decision making), dass der Patient seine „verlorengegangene" Ressource - das Schwimmen wiederentdeckt hat und diese Form der Bewegung trotz der Beschwerden wiederaufnimmt. Der Patient gibt an, wenn er im Wasser sei, gelinge es ihm, die Beschwerden zu vergessen und durch die Wiederaufnahme dieses Sports bessere sich die Symptomatik deutlich. Zudem knüpfte der Patient wieder soziale Kontakte im Rahmen einer wöchentlich stattfindenden Aqua-AerobicGruppe.

Schonung und Vermeidung können zwar kurzfristig die Ängste des Patienten mindern und die Arzt-Patient-Beziehung stabilisieren, sollten allerdings - entsprechend begründet - auch nur kurzfristig unterstützt werden.

Hilfreich ist es auch, auf geeignete Selbsthilfeliteratur und ggf. auf Selbsthilfegruppen zu verweisen. Insbesondere hilfreich sind auch Angebote außerhalb des Medizinsystems, z. B. an der Volkshochschule, die man nicht in einer Patientenrolle, sondern als Privatperson wahrnimmt.

\section{Zur körperlichen Aktivität ermutigen}

Körperliche Aktivierung ist eine zentrale Therapiemaßnahme bei funktionellen Körperbeschwerden. Aktivierung kann in Form kleiner Verhaltensveränderungen beginnen, die individuell angepasst sein sollten.

Dem Patienten sollte zu eigeninitiativer, genussvoller Bewegung geraten werden, egal in welchem Rahmen, mit welcher Methode oder Sportart. In diesem Zusammenhang sollte dem Patienten ebenfalls geraten werden, Phasen der Bewegung und körperlichen Anstren- 
gung mit Phasen der (ebenso genussvollen) Entspannung abzuwechseln.

Ein weiterer unterstützender Aspekt besteht in sozialer Aktivierung. Hierbei kann man dem Patienten zu Bewegungsaktivitäten in der Gruppe oder zu Mannschaftssportarten raten.

Zeitlich begrenzt lässt sich auch aktivierende Physiound Ergotherapie einsetzen.

Merke

Auf längerfristige Arbeitsunfähigkeitsbescheinigungen ist möglichst zu verzichten.

\section{PRAXISTIPP}

Schonung und Vermeidung sollten nur kurzfristig unterstützt und der Patient zu eigeninitiativer genussvoller Bewegung motiviert werden (Aktiv vor Passiv). Auf längerfristige Arbeitsunfähigkeitsbescheinigungen, vor allem ohne einen gemeinsam erarbeiteten (Therapie-)Plan für die Zeit der Arbeitsunfähigkeit (partizipative Entscheidungsfindung mit einer partnerschaftlichen Zusammenarbeit), sollte verzichtet werden.

\section{Zusammenarbeiten und Rat einholen}

Der Patient sollte nach parallel verlaufenden Behandlungen und Konsultationen sowie nach deren Wirkung gefragt und darum gebeten werden, dass der Behandler - bei Vorliegen einer Schweigepflicht-Entbindung sich mit den Kollegen der anderen Fachrichtungen austauschen darf.

Der Behandler sollte für eine gute Erreichbarkeit für Mitbehandler sorgen. Mit den Mitbehandlern sollte sich hinsichtlich diagnostischer Einordnung, eventueller Behandlungsprobleme und der weiteren Behandlungsplanung beraten werden. Bei besonders hart- näckigen Beschwerden und hoher Inanspruchnahme von Gesundheitsleistungen ist an mögliche aufrechterhaltende dysfunktionale Faktoren wie signifikante Vorteile durch die Krankenrolle, Medikamentenmissbrauch, gravierende Lebensereignisse oder an die Möglichkeit bisher unerkannter, eventuell seltener körperlicher/psychischer Differenzialdiagnosen zu denken und hier entsprechend der Rat von Kollegen einzuholen.

Merke

Auch Sie als Behandler sind nicht allein. Nutzen Sie Ihr Netzwerk und tauschen sich gegenseitig aus!

\section{Multimodale Behandlung, Psycho- therapie und Rehabilitation \\ Einbeziehung weiterer Behandler und Behandlungsformen}

Im Rahmen der multimodalen Behandlung liegt der Schwerpunkt auf der Einbindung von

- psychotherapeutischen sowie (teil)stationären Behandlungsformen sowie

- rehabilitativen Maßnahmen (> Abb.6).

\section{Multimodale Behandlung vorbereiten}

Bei der Vorbereitung einer multimodalen Behandlung sollte zunächst ein ambulantes Behandlernetzwerk angestrebt werden. Gegenseitige Kenntnis und Wertschätzung der Behandler und regelmäßige Kommunikation ist für eine gelingende multimodale Behandlung notwendig.

\section{Merke \\ Der primär behandelnde Arzt sollte weiterhin der Hauptansprechpartner für den Patienten und der Hauptverantwortliche für die Koordination der Be- handlung sein („Gatekeeper-Funktion“).}

Je nach diagnostischer Einschätzung, Motivation der Betroffenen und lokalen Gegebenheiten sollten auch eine andere somatisch-fachärztliche oder eine ambu-

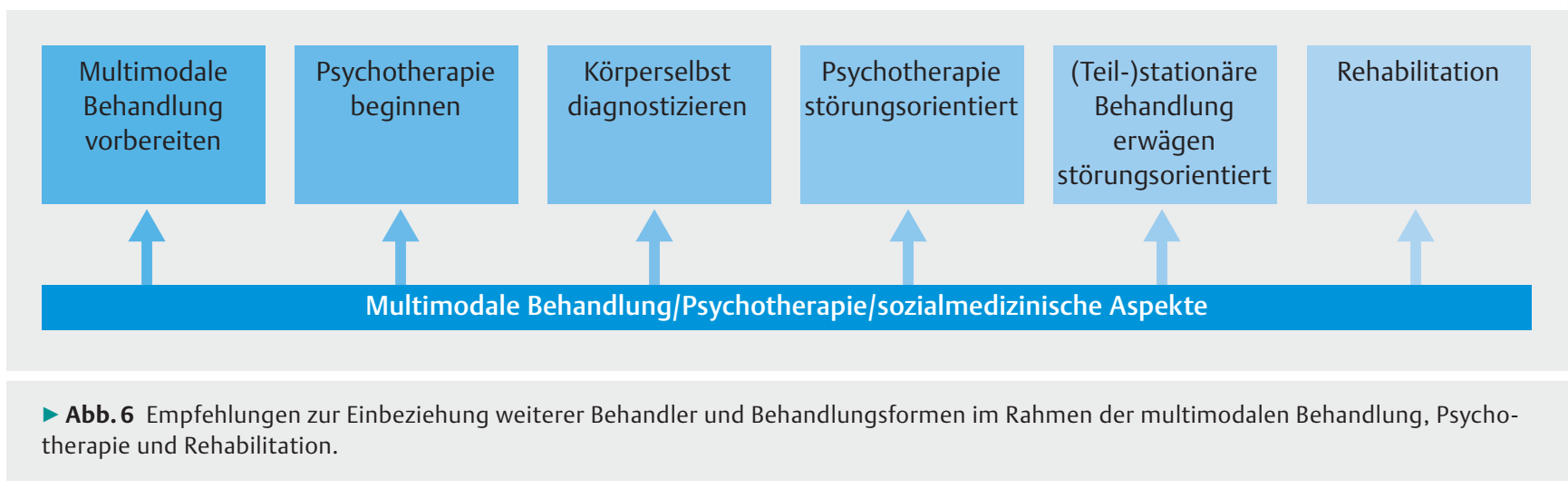


lante psychiatrische Mitbehandlung, eine begleitende Physiotherapie oder weitere Therapieelemente einbezogen werden.

Psychotherapie ist eine wichtige und bei einem breiten Spektrum funktioneller Körperbeschwerden nachweislich wirksame Behandlungsform, wenngleich nicht die einzige Option bei der Einbeziehung weiterer Behandlungskomponenten. Die Vermittlung in ambulante Psychotherapie sollte insbesondere erwogen werden bei:

- relevanten psychosozialen Belastungsfaktoren und/ oder psychischer Komorbidität

- relevanten dysfunktionalen Krankheitsannahmen

- starker funktioneller Beeinträchtigung

- anhaltend schwierigen Behandler-Patient-Beziehungen

Eventuell indizierte Über- oder Einweisungen, insbesondere zum Psychiater, Psychosomatiker und Psychotherapeuten, sollten behutsam vorbereitet werden. Die Sinnhaftigkeit dieses Vorgehens sollte dem Patienten erläutert werden und auch mögliche diesbezügliche Vorbehalte der Patienten sollten offen angesprochen werden.

Bei der Auswahl des psychotherapeutischen Verfahrens ist ein wissenschaftlich gut fundiertes Verfahren zu wählen, dessen Wirksamkeit durch qualitativ hochwertige Studien gesichert ist. Dies sind vor allem:

- (kognitive) Verhaltenstherapie

- psychodynamische Psychotherapie

- Hypnotherapie

Auch ergänzend zu einer Psychotherapie oder wenn diese trotz bestehender Indikation wegen lokaler Gegebenheiten, spezifischer/anders gerichteter Motivation von Patienten oder aus anderen Gründen nicht zustande kommt, sollten weitere Behandlungsformen erwogen werden, die sich im Rahmen multimodaler Behandlungsansätze bewährt haben:

- körperorientierte Verfahren wie beispielsweise Physiotherapie

- Entspannungsverfahren

- Bio-/Neuro-Feedback

- körpertherapeutische Verfahren (z. B. Feldenkrais, konzentrative Bewegungstherapie, Tanztherapie, Tai Chi, Qi-Gong)

- weitere nicht oder nur in weitem Sinne körperorientierte Verfahren (z. B. Gesundheitstraining, therapeutisches Schreiben, Musiktherapie, Yoga).
Keines dieser Verfahren ist als Monotherapie für eine adäquate Behandlung von chronischen und schweren Verläufen funktioneller Körperbeschwerden geeignet, sondern diese Therapieverfahren sind als Zusatzelemente im Rahmen einer multimodalen Therapie anzuwenden.

\section{PRAXISTIPP}

Auch in den spezifischen (Zusatz-)Behandlungen ist es wichtig, den Patienten in seiner Selbstwirksamkeit und Aktivierung zu fördern und die aktive Tätigkeit außerhalb der Krankenrolle zu fördern.

Eine möglichst zeitnahe Wiedervorstellung des Patienten beim Behandlungsführer sollte vereinbart werden, wenn eine Konsultation bei anderen Kollegen geplant oder bereits erfolgt ist.

\section{Psychotherapie beginnen}

Psychotherapien und psychiatrische Behandlungen verlassen das übliche, für viele Patienten vertraute Schema ärztlicher Konsultationen. Es sollte damit gerechnet werden, dass Patienten dieser ungewohnten Therapieform gegenüber zumindest ambivalent eingestellt sind. Psychotherapie sollte als wissenschaftlich begründete „Therapie mit psychologischen Mitteln“ erklärt werden, die auch bei körperlich definierten Beschwerden und Erkrankungen helfen kann. Psychotherapeuten werden hierfür „mit ins Boot“ geholt, Patienten werden nicht an diese abgegeben.

Merke

Motivation zur Psychotherapie kann ein erstes Therapieziel in der Psychotherapie sein („Motivationsaufbau“).

Vorerfahrungen, Erwartungen, Ängste und Vorbehalte des Patienten in Bezug auf das Medizinsystem, speziell auf Psychotherapie sollten erfragt werden. Hierbei ist erneut auf die in der Initialphase wichtigsten Behandlungselemente zu fokussieren:

- Vertrauensaufbau

- Psychoedukation

- Verbesserung konkreter Bewältigungsstrategien, wie z. B. Stress- und Schmerzbewältigung oder Akzeptanz

Die in der Grundversorgung empfohlenen Maßnahmen, z. B. zu Gesprächsführung, Formulierung von Zielen, Selbstwirksamkeit und Aktivierung, sollten ebenfalls zum Aufbau einer stabilen therapeutischen Arbeitsbeziehung genutzt werden. 
In der Therapie ist zunächst mit den Beschwerden, beschwerdebezogenen Einstellungen und Verhaltensweisen sowie mit dem bisherigen Erklärungsmodell des Patienten zu arbeiten. Psychologische Zusammenhänge der Beschwerdeentstehung und Aufrechterhaltung sollten initial eher „tangential“, also nicht zu direkt, eingebracht werden.

\section{Körperselbst diagnostizieren}

Die Initialphase der Psychotherapie sollte für eine umfassendere Diagnostik des Erlebens und Verhaltens von Patienten mit funktionellen Körperbeschwerden genutzt werden - parallel zum Aufbau der therapeutischen Arbeitsbeziehung (s. o.).

In der erweiterten Psychodiagnostik sollte hier auch insbesondere Berücksichtigung finden:

- Dysfunktionale beschwerdebezogene Denk- und Verhaltensweisen

- Erfahrungen mit eigener Krankheit

- Erfahrungen mit Rollenmodellen (vermeintlich) schwerer Erkrankungen in Familie und Umfeld (z. B. Erkrankung eines Elternteils)

- Mögliche Traumata, Gewalt- und Vernachlässigungserfahrungen

- Das Vorliegen weiterer möglicher krankheitsaufrechterhaltender Faktoren, wie z. B. aktuelle Belastungen und Konflikte, Berentungswunsch oder in Aussicht stehende Kompensationsleistungen.

- Psychische Komorbiditäten, insbesondere aus dem Bereich der Angst- und affektiven Erkrankungen, der Traumafolgestörungen, der Suchterkrankungen und der Persönlichkeitsstörungen.

\section{Psychotherapie - störungsorientiert}

In der Psychotherapie sollte ein störungsorientiertes und wissenschaftlich gut fundiertes psychotherapeutisches Verfahren angewandt werden.

Vulnerable Themen können angesprochen werden, wenn der Patient selbst Interesse für psychosoziale Themen zeigt oder im Therapieverlauf erlebt, dass seine Beschwerden durch kleine Änderungen von Erwartungen und Verhalten beeinflussbar sind. In der Hauptphase der Therapie sind mögliche Schwerpunkte auf folgende Aspekte zu legen:

- positives Selbst- und Körpererleben

- Selbstregulationstechniken

- Beziehungsgestaltung

- Förderung von Kreativität und Veränderungsbereitschaft

\section{(Teil-)stationäre Behandlung erwägen}

Sollte keine ambulante Behandlung möglich sein oder sich als unzureichend erweisen, ist eine multimodale Behandlung in einer geeigneten (teil-)stationären Einrichtung zu erwägen. Diese soll eine interdisziplinäre Behandlung nach abgestimmtem Gesamtbehandlungsplan unter qualifizierter ärztlicher Leitung anbieten.

Der Behandlungsplan sollte mehrere therapeutische Angebote systematisch kombinieren:

- organbezogene/symptomatische Therapien

- Psychotherapie (möglichst sowohl im Einzelsetting als auch in Gruppen)

- körperbezogene Therapie und Physiotherapie, sowie weitere therapeutische Angebote.

Eine stationäre Behandlung in einer Klinik mit multimodalem Therapiekonzept sollte vor allem bei folgenden Indikationen erwogen werden:

- Schwere der körperlichen und/oder psychischen Symptomatik bzw. der funktionellen Beeinträchtigung

- Chronifizierungsgefahr

- krisenhafte Zuspitzung

- Erfolglosigkeit der ambulanten Therapie

- Nichtverfügbarkeit einer ambulanten multimodalen Behandlung

- relevante sozialmedizinische Aspekte

- ausgeprägte interaktionelle Probleme in der Behandler-Patient-Beziehung

\section{Rehabilitation}

Als Behandlungsoption für Patienten mit funktionellen Körperbeschwerden kann auch eine medizinische Rehabilitation erwogen werden, insbesondere wenn der Fokus darauf liegt, die Teilhabe zu verbessern einschließlich des Erhalts oder der Wiederherstellung der Erwerbsfähigkeit und damit eine (weitere) Chronifizierung zu verhindern.

\footnotetext{
Merke

Rehabilitationsbehandlungen sind fachübergreifend anzulegen, mit einem ausreichenden Anteil an Beratung, Psychodiagnostik und Psychotherapie.
} 


\section{KERNAUSSAGEN}

- Körperbeschwerden sind häufig, präsentieren sich mit unterschiedlichen Symptomen und sind in ihrer Ausprägung bzw. in ihrem Schweregrad verschiedenartig.

- Die Prävalenz von funktionellen Körperbeschwerden in der Allgemeinbevölkerung wird mit etwa $10 \%$ angegeben; im hausärztlichen Bereich liegt diese bei $20-50 \%$ und in spezialisierten Settings zwischen 25 und 66\%.

- Ätiologisch geht man bei funktionellen Körperbeschwerden von einer multifaktoriellen Genese aus, die aus verschiedenen und unterschiedlich gewichteten biopsychosozialen Faktoren besteht.

- Die Einordnung „funktionell“ ist bei den ersten Präsentationen oftmals eine Herausforderung; sie sollte jedoch von Anfang an - gleichwertig wie potenzielle abwendbar gefährliche Verläufe der Beschwerden - mitgedacht werden.

- Die initiale Grundversorgung besteht darin, funktionelle Körperbeschwerden zu bemerken und zu erkennen und den Patienten mit Zurückhaltung, Wachsamkeit und Empathie zu begegnen.

- Die erweiterte Grundversorgung bezieht sich anfangs auf den bewussten weiteren Umgang mit Patienten (Simultandiagnostik sowohl somatischer als auch psychosozialer Anteile).

- Der weitere Behandlungsverlauf besteht im Wesentlichen aus der Vermittlung eines nachvollziehbaren, für den Patienten akzeptablen Erklärungsmodells und der Stärkung von Selbstwirksamkeit.

- Im Rahmen der multimodalen Behandlung liegt der Schwerpunkt auf der Einbindung von psychotherapeutischen sowie (teil)stationären Behandlungsformen sowie ggf. von rehabilitativen Maßnahmen.

\section{Schlüsselwörter}

Funktionelle Körperbeschwerden, Somatische Belastungsstörung, Somatoforme Störung

\section{Interessenkonflikt}

\section{Erklärung zu finanziellen Interessen}

Forschungsförderung erhalten: nein; Honorar/geldwerten Vorteil für Referententätigkeit erhalten: ja; Bezahlter Berater/interner Schulungsreferent/Gehaltsempfänger: nein; Patent/Geschäftsanteile/Aktien (Autor/Partner, Ehepartner, Kinder) an Firma (Nicht-Sponsor der Veranstaltung): ja; Patent/Geschäftsanteile/Aktien (Autor/Partner, Ehepartner, Kinder) an Firma (Sponsor der Veranstaltung): nein.

\section{Erklärung zu nichtfinanziellen Interessen}

Oberarzt der Station N1a der Klinik für Psychosomatik und Psychotherapie am Klinikum rechts der Isar der Technischen Universität München

Bereichsleitender Oberarzt Psychosomatik im Zentrum für interdisziplinäre Schmerztherapie am Klinikum rechts der Isar der Technischen Universität München
Mitglied der Deutschen Gesellschaft für Psychosomatische Medizin

Mitglied der Deutschen Gesellschaft für Allgemeinmedizin

\section{Autorinnen/Autoren}

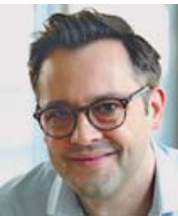

\section{Casper J. Roenneberg}

Dr. med., MHBA. 2003-2009 Studium der Humanmedizin in Berlin und Chicago, USA. 2013-2015 berufsbegleitender Fernstudiengang „Master of Health Business Administration (MHBA)" an der Friedrich-Alexander-Universität Erlangen-Nürnberg. 2010-2015 Arzt in Weiterbildung Chirurgie, Innere Medizin und Allgemeinmedizin. Facharzt für Allgemeinmedizin 2015. Seit 2015 Arzt und wissenschaftlicher Mitarbeiter der Klinik für Psychosomatische Medizin und Psychotherapie des Klinikums rechts der Isar; seit 04/2018 Oberarzt der Klinik für Psychosomatische Medizin und Psychotherapie und im Zentrum für Interdisziplinäre Schmerztherapie des Klinikums rechts der Isar der TU München.

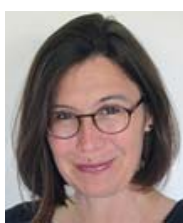

\section{Constanze Hausteiner-Wiehle}

Prof. Dr. med. 1991-1997 Studium der Humanmedizin in München (LMU), Witten/Herdecke, Zürich, Cleveland und Wuppertal. 1998-1999 Ärztin im Praktikum Klinik für Neurologie Wuppertal; 1999-2007 Klinik und Poliklinik für Psychiatrie und Psychotherapie und Toxikologischen Abteilung der II. Medizinischen Klinik und Klinik der TU München. Seit 2007 Ärztin und wissenschaftliche Mitarbeiterin der Klinik und Poliklinik für Psychosomatische Medizin und Psychotherapie der TU München. Seit 2012 Psychosomatischer Konsil- und Liaisondienst in der BG Unfallklinik Murnau.

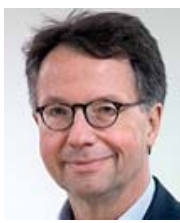

\section{Peter Henningsen}

Professor Dr. med. 1977-1984 Studium der Humanmedizin in Stuttgart-Hohenheim, Freiburg, Berlin und Cambridge, UK. 19841990 Oberarzt Kliniken der Neurologie der Freien Universität Berlin und der Universität Heidelberg. 1990-2005 Oberarzt und leitender Oberarzt der Psychosomatischen Klinik der Universität Heidelberg; Facharzt für Neurologie und Psychiatrie (1995), Facharzt für Psychosomatische Medizin und Psychotherapie (1997). Seit 2005 Direktor und Lehrstuhlinhaber, Klinik für Psychosomatische Medizin und Psychotherapie und Dekan der Fakultät für Medizin der TU München.

\section{Korrespondenzadresse}

\section{Dr. med. Casper J. Roenneberg, MHBA}

Klinik und Poliklinik für Psychosomatische Medizin und Psychotherapie

Klinikum rechts der Isar der Technischen Universität München

Ismaninger Str. 22

81675 München

E-Mail: Casper.Roenneberg@mri.tum.de 
Wissenschaftlich verantwortlich gemäß Zertifizierungsbestimmungen

Wissenschaftlich verantwortlich gemäß Zertifizierungsbestimmungen für diesen Beitrag ist Dr. med. Casper J. Roenneberg, München.

\section{Literatur}

[1] Hiller W, Rief W, Brahler E. Somatization in the population: from mild bodily misperceptions to disabling symptoms. Soc Psychiatry Psychiatr Epidemiol 2006; 41: 704-712

[2] Henningsen P, Zipfel S, Sattel H et al. Management of functional somatic syndromes and bodily distress. Psychother Psychosom 2018; 87: 12-31

[3] Gierk B, Kohlmann S, Kroenke K et al. The somatic symptom scale-8 (SSS-8): a brief measure of somatic symptom burden. JAMA Intern Med 2014; 174: 399-407

[4] WHO. ICD-11 for Mortality and Morbidity Statistics (Version: 04/2019). Geneva: World Health Organisation (WHO); 2019

[5] Deutsche Gesellschaft für Psychosomatische Medizin und Ärztliche Psychotherapie e.V. (DGPM), DKfPMD. u. a. S3 Leitlinie „Funktionelle Körperbeschwerden“. Arbeitsgemeinschaft der Wissenschaftlichen Medizinischen Fachgesellschaften e.V. (AWMF) e.V. 2018

[6] Hilderink PH, Collard R, Rosmalen JG et al. Prevalence of somatoform disorders and medically unexplained symptoms in old age populations in comparison with younger age groups: a systematic review. Ageing Res Rev 2013; 12: 151 156

[7] Nimnuan C, Hotopf M, Wessely S. Medically unexplained symptoms: an epidemiological study in seven specialities. J Psychosom Res 2001; 51: 361-367

[8] Snijders T], de Leeuw FE, Klumpers UM et al. Prevalence and predictors of unexplained neurological symptoms in an academic neurology outpatient clinic-an observational study. J Neurol 2004; 251: 66-71
[9] Maiden NL, Hurst NP, Lochhead A et al. Medically unexplained symptoms in patients referred to a specialist rheumatology service: prevalence and associations. Rheumatology (Oxford) 2003; 42: 108-112

[10] de Waal MW, Arnold IA, Eekhof JA et al. Somatoform disorders in general practice: prevalence, functional impairment and comorbidity with anxiety and depressive disorders. $\mathrm{Br}$ J Psychiatry 2004; 184: 470-476

[11] Budtz-Lilly A, Vestergaard M, Fink P et al. The prognosis of bodily distress syndrome: a cohort study in primary care. Gen Hosp Psychiatry 2015; 37: 560-566

[12] Jackson JL, Kroenke K. Prevalence, impact, and prognosis of multisomatoform disorder in primary care: a 5 -year followup study. Psychosom Med 2008; 70: 430-434

[13] Steinbrecher N, Hiller W. Course and prediction of somatoform disorder and medically unexplained symptoms in primary care. Gen Hosp Psychiatry 2011; 33: 318-326

[14] Lieb R, Zimmermann P, Friis RH et al. The natural course of DSM-IV somatoform disorders and syndromes among adolescents and young adults: a prospective-longitudinal community study. Eur Psychiatry 2002; 17: 321-331

[15] olde Hartmann TC, Borghuis MS, Lucassen PL et al. Medically unexplained symptoms, somatisation disorder and hypochondriasis: course and prognosis. A systematic review. J Psychosom Res 2009; 66: 363-377

[16] Hatcher S, Gilmore K, Pinchen K. A follow-up study of patients with medically unexplained symptoms referred to a liaison psychiatry service. Int J Psychiatry Med 2011; 41: 217-227

[17] Eikelboom EM, Tak LM, Roest AM et al. A systematic review and meta-analysis of the percentage of revised diagnoses in functional somatic symptoms. J Psychosom Res 2016; 88: 60-67

[18] Riess H, Kraft-Todd G. E.M.P.A.T.H.Y.: a tool to enhance nonverbal communication between clinicians and their patients. Acad Med 2014; 89: 1108-1112

[19] Burton C, Lucassen P, Aamland A et al. Explaining symptoms after negative tests: towards a rational explanation. J R Soc Med 2015; 108: 84-88

Bibliografie

DOI https://doi.org/10.1055/a-0901-5103

PSYCH up2date 2020; 14: 35-53

(c) Georg Thieme Verlag KG Stuttgart · New York ISSN 2194-8895 


\section{Punkte sammeln auf CME.thieme.de}

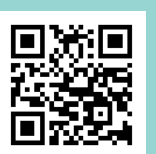

Diese Fortbildungseinheit ist in der Regel 12 Monate online für die Teilnahme verfügbar.

Den genauen Einsendeschluss finden Sie unter https://cme.thieme.de/CXD1EK7.

Sollten Sie Fragen zur Online-Teilnahme haben, finden Sie unter https://cme.thieme.de/hilfe

eine ausführliche Anleitung. Wir wünschen viel Erfolg beim Beantworten

der Fragen!

Unter https://eref.thieme.de/CXD1EK7 oder über den QR-Code kommen Sie direkt zur Startseite des Wissenstests.

VNR 2760512020158722773

\section{Frage 1}

Wie hoch ist die Prävalenz von Menschen mit funktionellen Körperbeschwerden in der Allgemeinbevölkerung?
A $1 \%$
B $5 \%$
C $10 \%$
D $20 \%$
E $50 \%$

\section{Frage 2}

Wie hoch ist die Prävalenz von Patienten mit funktionellen Körperbeschwerden in hausärztlichen Primärversorgung?
A zwischen 5 und $10 \%$
B zwischen 10 und $20 \%$
C zwischen 15 und $30 \%$
D zwischen 25 und $40 \%$
E zwischen 20 und $50 \%$

\section{Frage 3}

Wie hoch ist die Prävalenz von Patienten mit funktionellen Körperbeschwerden in spezialisierten Settings, wie beispielsweise rheumatologischen, gynäkologischen oder schmerzmedizinischen Ambulanzen?
A zwischen 15 und $30 \%$
B zwischen 20 und $40 \%$
C zwischen 25 und $50 \%$
D zwischen 25 und $66 \%$
E zwischen 30 und $72 \%$

\section{Frage 4}

Wie hoch ist in etwa die Rate fälschlich als „funktionell“ eingeordneter Beschwerden?
A $\quad 0,1-1 \%$
B $\quad 0,2-5,5 \%$
C $0,5-8,8 \%$
D $1-10 \%$
E $5-25 \%$

\section{Frage 5}

Welche Faktoren sind keine Schutzfaktoren für einen günstigen Verlauf von funktionellen Körperbeschwerden?
A funktionale Gedanken und Einstellungen
B aktive Bewältigungsstrategien
C passives Patientenverhalten
D individuelle Ressourcen
E tragfähige Arzt-Patient-Beziehung

\section{Frage 6}

Welche Faktoren sind keine Indikatoren/Risikofaktoren für einen schweren Verlauf von funktionellen Körperbeschwerden?
A mehrere Beschwerden (polysymptomatischer Verlauf)
B häufige bzw. anhaltende Beschwerden
C dysfunktionale Gedanken und Einstellungen
D psychische Komorbidität
E biopsychosozialer Behandlungsansatz

\section{Frage 7}

Welche Empfehlung zählt nicht zu der initialen Grundversorgung von Patienten mit funktionellen Körperbeschwerden?
A Integration eines störungsorientierten Psychotherapiever- fahrens
B Beruhigen
C Beraten
D Abwartendes Offenhalten
E Sorgfältig befragen und untersuchen

\section{Frage 8}

Welchen Aspekt sollte ein gutes Erklärungsmodell für funktionelle Körperbeschwerden beinhalten?
A immer Ursachen der Beschwerden nennen können
B immer eine Diagnose beinhalten
C vor allem für den Behandler plausibel erscheinen
D keine Schwäche oder Schuld beim Patienten implizieren
E ohne Rücksicht auf die therapeutische Beziehung ent- worfen werden 


\section{Punkte sammeln auf CME.thieme.de}

Fortsetzung ...

\section{Frage 9}

Was versteht man unter einem „Sowohl-als-auch“-Ansatz?

A die Integration von Anamnese und körperlicher Untersuchung in einen diagnostischen Schritt

B das gemeinsame Vorliegen von psychischen Diagnosen (z.B. Depression) als auch einer chronischen somatischen Erkrankung (z. B. Herzinsuffizienz)

C eine zurückhaltende Einordnung der Beschwerdepräsentation mit dem fortwährenden Antizipieren von sowohl psychosozialen Faktoren als auch abwendbar gefährlichen Verläufen und Chronifizierungsfaktoren von somatischen Erkrankungen

D das Vorliegen sowohl einer Funktionsstörung als auch einer Funktionalität einer Störung (z. B. vermehrte Zuwendung von Angehörigen)

E die gemeinsame Behandlung von Patienten mit funktionellen Körperbeschwerden sowohl durch einen somatischen Arzt als auch einen Psychiater oder psychologischen Psychotherapeuten

\section{Frage 10}

Wann sollte eine stationäre Therapie von Patienten mit funktionellen Körperbeschwerden erwogen werden?

A so früh wie möglich im Behandlungsverlauf

B schon bei leichten körperlichen Einschränkungen eines Organsystems ohne relevante Beeinträchtigung

C im Rahmen einer „Kur“ für jeden Patienten - auch bei guten ambulanten Behandlungsbedingungen

D bei Chronifizierungsgefahr

E vor Beginn einer ambulanten Psychotherapie 